
COMMUNICATIONS

ISSN 2056-9890
CRYSTALLOGRAPHIC

\section{Crystal structure and Hirshfeld surface analysis of 1-[(1-butyl-1H-1,2,3-triazol-4-yl)methyl]-3-methyl- 1,2-dihydroquinoxalin-2-one}

\author{
Nadeem Abad, ${ }^{a *}$ Youssef Ramli, ${ }^{b}$ Tuncer Hökelek, ${ }^{c}$ Nada Kheira Sebbar, ${ }^{\text {d }}$ Joel T. \\ Mague $^{\mathrm{e}}$ and El Mokhtar Essassi ${ }^{\mathrm{a}}$
}

Received 25 October 2018

Accepted 9 November 2018

Edited by D.-J. Xu, Zhejiang University (Yuquan Campus), China

Keywords: crystal structure; dihydroquinoxaline; triazole; $\pi$-stacking; Hirshfeld surface.

CCDC reference: 1878133

Supporting information: this article has supporting information at journals.iucr.org/e

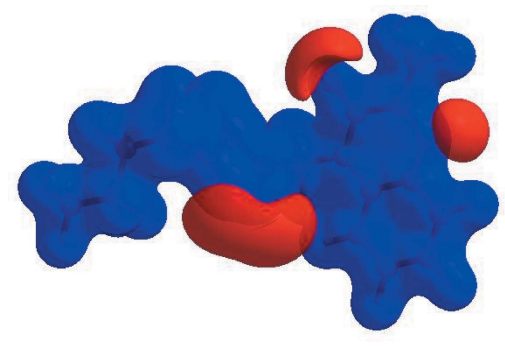

\section{Chemical context}

Quinoxaline groups are well known, important nitrogencontaining heterocyclic compounds comprising a benzene and a pyrazine ring fused together. Diversely substituted quinoxalines and their derivatives embedded with variety of functional groups are important biological agents and a significant amount of research activity has been directed towards this class of compounds. These molecules exhibit a wide range of biological applications and are potentially useful in medicinal chemistry research and have therapeutic applications such as antimicrobial (Attia et al., 2013; Vieira et al., 2014; Teja et al., 2016), anti-inflammatory (Guirado et al., 2012), anticancer (Abbas et al., 2015), antidiabetic (Kulkarni et al., 2012) and antihistaminic activities (Sridevi et al., 2010). As a continuation of our research works on the synthesis, spectroscopic and biological properties of quinoxaline derivatives (Ramli et al., 2013, 2017; Ramli \& Essassi, 2015; Abad et al., 2018a,b,c; Ellouz et al., 2015; Sebbar et al., 2014), we report herein the molecular and crystal structures along with the Hirshfeld surface analysis of the title compound, 1-[(1-butyl- $1 H-1,2,3-$ triazol-5-yl)methyl]-3-methyl-1,2-dihydroquinoxalin-2-one.

\section{Structural commentary}

The title compound, (I), is built up from the two fused sixmembered rings of a quinoxalinone moiety linked through a 
methylene bridge to a 1,2,3-triazole ring, which in turn carries an $n$-butyl substituent on N3 (Fig. 1). The dihydroquinoxaline unit is planar within 0.029 (1) $\AA$ (r.m.s. deviation of the fitted atoms $=0.0123 \AA$ ) and the triazole ring is inclined by $67.09(4)^{\circ}$ to the above-mentioned plane. The molecule adopts a Z-shaped conformation with the (1H-1,2,3-triazol-5-yl)methyl substituent projecting well out of the mean plane of the dihydroquinxalone unit, as indicated by the $\mathrm{C} 1-\mathrm{N} 2-\mathrm{C} 10-$ C11 torsion angle of $90.85(16)^{\circ}$. The $n$-butyl group is oriented in the opposite direction as seen from the N4-N3-C13-C14 torsion angle of $-95.26(16)^{\circ}$ (Fig. 2).

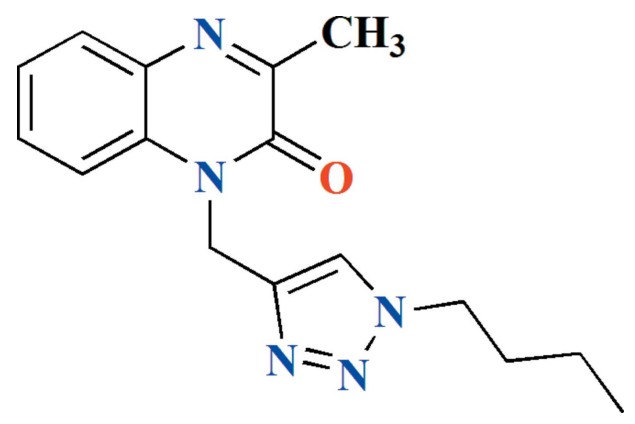

\section{Supramolecular features}

Hydrogen bonding and van der Waals contacts are the dominant interactions in the crystal packing. In the crystal, the molecules form oblique stacks along the $a$-axis direction through intermolecular $\mathrm{C}-\mathrm{H}_{\mathrm{Trz}} \cdots \mathrm{N}_{\mathrm{Trz}}$ ( $\mathrm{Trz}=$ triazole) hydrogen bonds (Table 1 ), and offset, very weak $\pi$-stacking interactions between the $A(\mathrm{C} 1-\mathrm{C} 6)$ and $B(\mathrm{~N} 1 / \mathrm{N} 2 / \mathrm{C} 1 / \mathrm{C} 6-\mathrm{C} 8)$ rings [centroid-centroid distance $=3.9107(9) \AA$, dihedral angle $\left.=0.94(7)^{\circ}\right]$ and $\pi$-interactions between the $\mathrm{C} 8=\mathrm{O} 1$ carbonyl group and the $B$ rings $[\mathrm{O} 1-$ centroid =

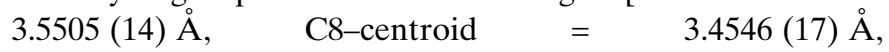
$\mathrm{C} 8=\mathrm{O} 1 \cdots$ centroid $\left.=75.51(9)^{\circ}\right]$. Pairs of stacks are associated through $\mathrm{C}-\mathrm{H}_{\text {Dhydqn }} \cdots \pi$ (Dhydqn $=$ dihydroquinoxaline) interactions, generating small, diamond-shaped channels along the $a$-axis direction (Table 1 and Fig. 2).

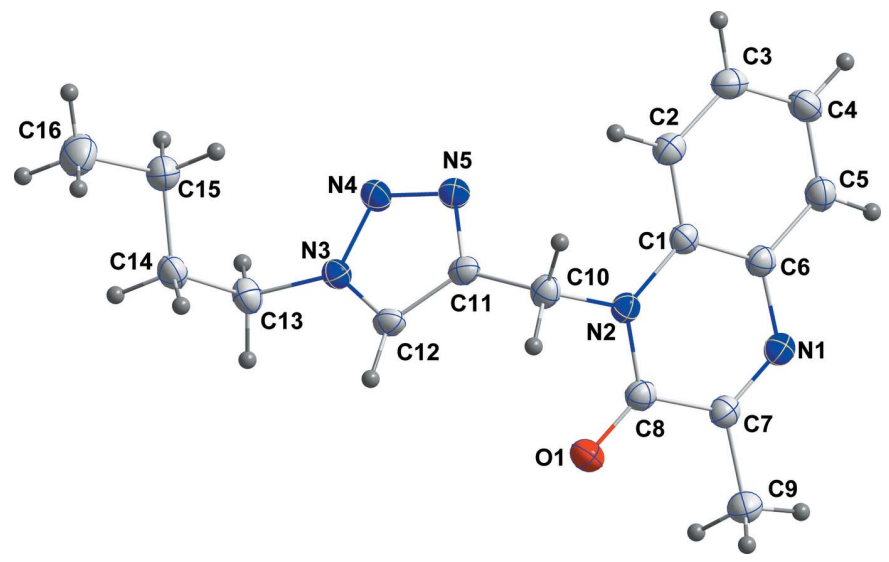

Figure 1

The title molecule with labelling scheme and $50 \%$ probability ellipsoids.
Table 1

Hydrogen-bond geometry $\left(\AA,^{\circ}\right)$.

$C g 1$ is the centroid of the $\mathrm{N} 3-\mathrm{N} 5 / \mathrm{C} 11 / \mathrm{C} 12$ ring.

\begin{tabular}{lllll}
\hline$D-\mathrm{H} \cdots A$ & $D-\mathrm{H}$ & $\mathrm{H} \cdots A$ & $D \cdots A$ & $D-\mathrm{H} \cdots A$ \\
\hline $\mathrm{C} 12-\mathrm{H} 12 \cdots \mathrm{N} 4^{\mathrm{iii}}$ & $0.954(19)$ & $2.419(19)$ & $3.2282(19)$ & $142.4(15)$ \\
$\mathrm{C} 2-\mathrm{H} 2 \cdots C g 1^{\mathrm{vii}}$ & $0.966(18)$ & $2.986(19)$ & $3.642(1)$ & $126.3(14)$ \\
\hline
\end{tabular}

Symmetry codes: (iii) $x-1, y, z$; (vii) $-x+1,-y+1,-z+1$.

\section{Database Survey}

A search of the CSD (Version 5.39, updated May 2018; Groom et al., 2016) using the fragment shown in Scheme $2(R=\mathrm{C}, R 1$ $=$ nothing) generated 37 hits. Of these, the ones most comparable to the title molecule have $R 1=\mathrm{CH}_{3}$ and $R=$ $\mathrm{CH}_{2} \mathrm{C} \equiv \mathrm{CH}$ (Benzeid et al., 2009), $\mathrm{CH}_{2} \mathrm{Ph}$ (Ramli et al., 2010a, 2018), $\mathrm{C}_{2} \mathrm{H}_{5}$ (Benzeid et al., 2008), (1,3-oxazolidin-3-yl)ethyl (Caleb et al., 2009), $\mathrm{CH}_{2} \mathrm{CH}=\mathrm{CH}_{2}$ (Ramli et al., 2010b) and the isomer with $R=$ (1-butyl-1H-1,2,3- triazol-5-yl)methyl (Abad et al., 2018a). Those with $R=\mathrm{CH}_{2} \mathrm{C} \equiv \mathrm{CH}$ and $\mathrm{C}_{2} \mathrm{H}_{5}$ have $Z^{\prime}=1$. A common feature of the above subset as well as the majority of the other compounds with different $R 1$ substituents is the geometry of the bicyclic unit, which is either planar or has a slight end-to-end twist. Another feature is the orientation of the $R$ group, which generally has a $\mathrm{C}-\mathrm{N}-\mathrm{C}-$ $\mathrm{C}$ torsion angle $>65^{\circ}$ and in quite a few cases, this is close to $90^{\circ}$. A comparison of the conformation of the title molecule with that of its (1-butyl-1H-1,2,3-triazol-5-yl)methyl isomer shows that the latter has a $\mathrm{U}$ shape with the $R$ group extending back over the bicyclic unit as the result of an intramolecular $\mathrm{C}-\mathrm{H}$... O hydrogen bond from the $\alpha$ hydrogen of the butyl group while in the former, the more remote position of the butyl group on the triazole ring disfavours such an interaction and the molecule adopts a $\mathrm{Z}$ shape. This conformation is favoured by the opportunity for $\pi$-stacking and $\mathrm{C}$ $\mathrm{H} \cdots \pi$ (ring) interactions in the crystal.

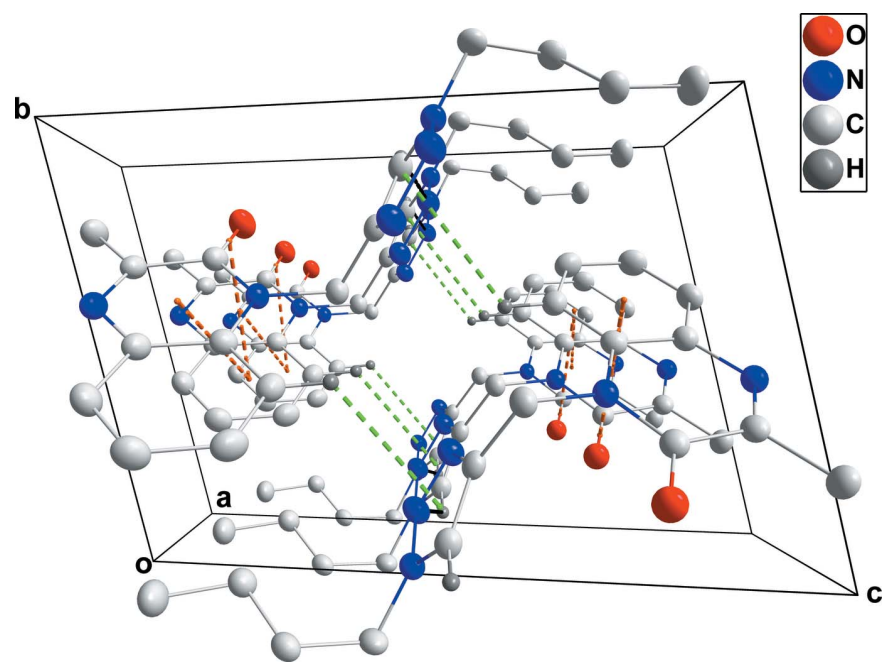

Figure 2

Packing viewed along the $a$-axis direction. $\mathrm{C}-\mathrm{H} \cdots \mathrm{N}$ hydrogen bonds are shown by black dashed lines while $\pi$-stacking and $\mathrm{C}-\mathrm{H} \cdots \pi$ (ring) interactions are shown, respectively, by orange and green dashed lines. 


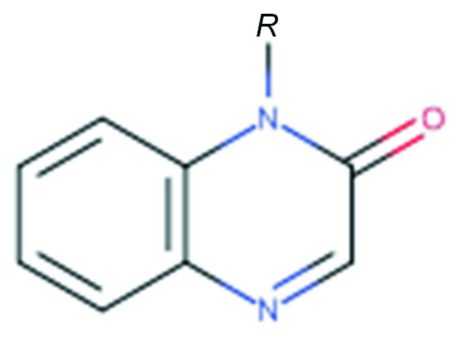

\section{Hirshfeld surface analysis}

In order to visualize the intermolecular interactions in the crystal of the title compound, a Hirshfeld surface (HS) analysis (Hirshfeld, 1977; Spackman \& Jayatilaka, 2009) was carried out using CrystalExplorer17.5 (Turner et al., 2017). In the HS plotted over $d_{\text {norm }}$ (Fig. 3), the white surface indicates contacts with distances equal to the sum of van der Waals radii, and the red and blue colours indicate distances shorter (in close contact) or longer (distant contact) than the sum of the van der Waals radii, respectively (Venkatesan et al., 2016). The bright-red spots appearing near the hydrogen atom H12 indicates their role as the respective donors and/or acceptors in the dominant $\mathrm{C}-\mathrm{H} \cdots \mathrm{N}$ hydrogen bonds; they also appear as blue and red regions corresponding to positive and negative potentials on the HS mapped over electrostatic potential (Spackman et al., 2008; Jayatilaka et al., 2005) as shown in Fig. 4. The blue regions indicate positive electrostatic potential (hydrogen-bond donors), while the red regions indicate negative electrostatic potential (hydrogen-bond acceptors). The shape-index of the HS is a tool to visualize the $\pi-\pi$ stacking by the presence of adjacent red and blue triangles; if there are no adjacent red and/or blue triangles, then there are no $\pi-\pi$ interactions. Fig. 5 clearly suggest that there are $\pi-\pi$ interactions in (I). The overall two-dimensional fingerprint plot, Fig. 6(a), and those delineated into $\mathrm{H} \cdots \mathrm{H}, \mathrm{H} \cdots \mathrm{N} / \mathrm{N} \cdots \mathrm{H}$, $\mathrm{H} \cdots \mathrm{C} / \mathrm{C} \cdots \mathrm{H}, \mathrm{H} \cdots \mathrm{O} / \mathrm{O} \cdots \mathrm{H}, \mathrm{C} \cdots \mathrm{C}, \mathrm{O} \cdots \mathrm{C} / \mathrm{C} \cdots \mathrm{O}, \mathrm{N} \cdots \mathrm{C} /$ $\mathrm{C} \cdots \mathrm{N}$ and $\mathrm{N} \cdots \mathrm{N}$ contacts (McKinnon et al., 2007) are illus-

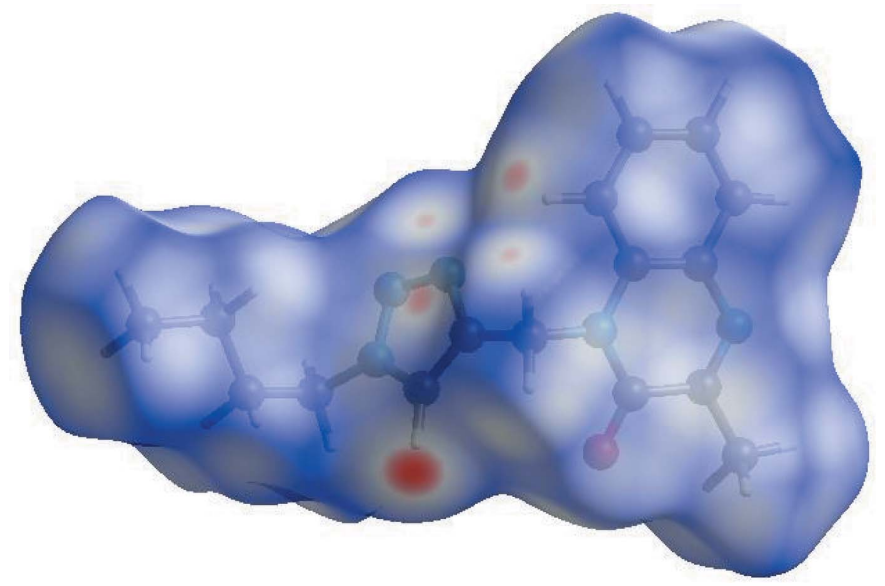

Figure 3

View of the three-dimensional Hirshfeld surface of the title compound plotted over $d_{\text {norm }}$ in the range -0.2380 to 1.1723 a.u.

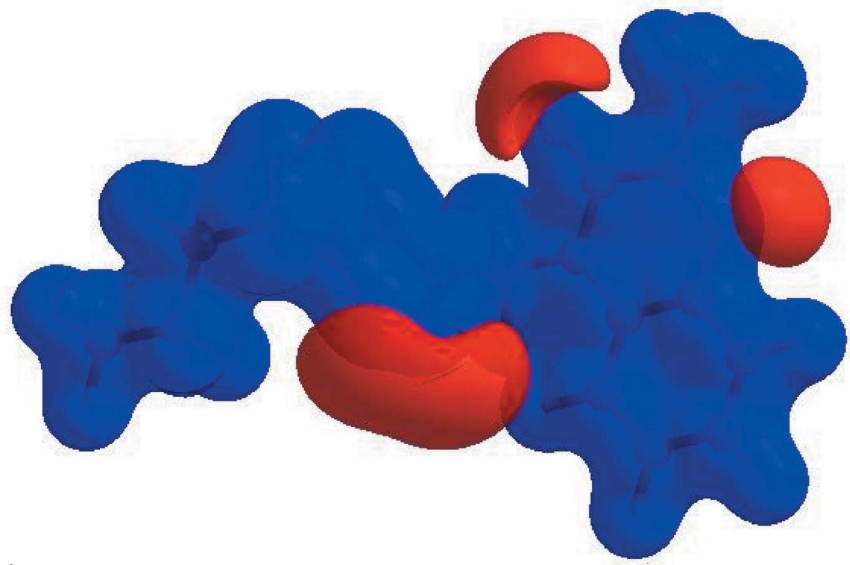

Figure 4

View of the three-dimensional Hirshfeld surface of the title compound plotted over electrostatic potential energy in the range -0.0500 to 0.0500 a.u. using the STO-3 G basis set at the Hartree-Fock level of theory. Hydrogen-bond donors and acceptors are shown as blue and red regions around the atoms corresponding to positive and negative potentials, respectively.

trated in Fig. 6(b)-(i), respectively, together with their relative contributions to the Hirshfeld surface. The most important interaction is $\mathrm{H} \cdots \mathrm{H}$ contributing $52.7 \%$ to the overall crystal packing, which is reflected in Fig. $6(b)$ as widely scattered points of high density due to the large hydrogen content of the molecule. The split spike with the tip at $d_{\mathrm{e}}=d_{\mathrm{i}}=1.13 \AA$ in Fig. 6(b) is due to the short interatomic $\mathrm{H} \cdots \mathrm{H}$ contacts (Table 2). The pair of characteristic wings resulting in the fingerprint plot delineated into $\mathrm{H} \cdots \mathrm{N} / \mathrm{N} \cdots \mathrm{H}$ contacts Fig. $6(c)$, contribute $18.9 \%$ to the HS (Table 2) and are viewed as pair of spikes with the tips at $d_{\mathrm{e}}+d_{\mathrm{i}}=2.23 \AA$. In the presence of weak $\mathrm{C}-\mathrm{H} \cdots \pi$ interactions (Table 1) in the crystal, the pair of characteristic wings resulting in the fingerprint plot delineated into $\mathrm{H} \cdots \mathrm{C} / \mathrm{C} \cdots \mathrm{H}$ contacts with a $17.0 \%$ contribution to the HS have a symmetrical distribution of points, Fig. $7(d)$, with the tips at $d_{\mathrm{e}}+d_{\mathrm{i}}=2.65 \AA$ (Table 2). Finally, the $\mathrm{H} \cdots \mathrm{O} / \mathrm{O} \cdots \mathrm{H}$ [Fig. 6(e)] contacts (Table 2) in the structure with $6.8 \%$ contribution to the HS also have

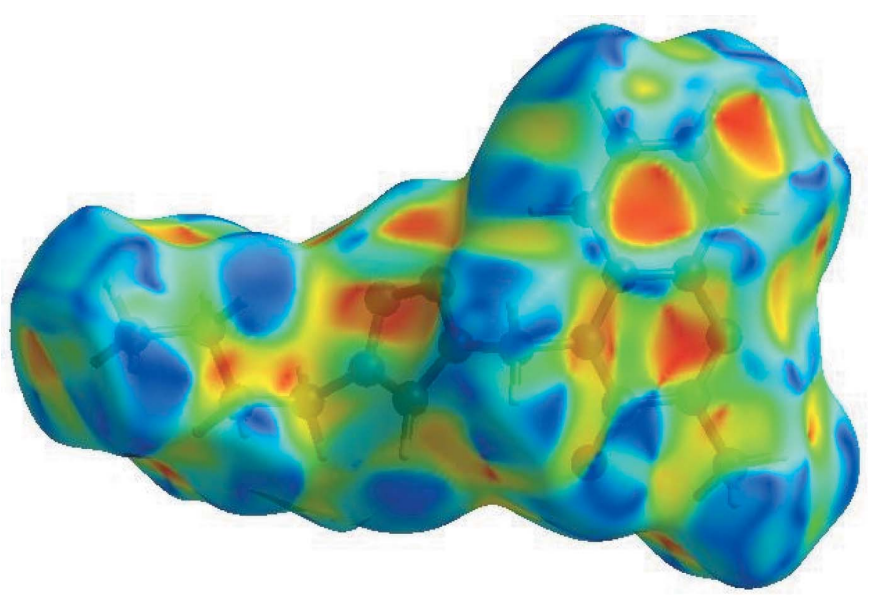

Figure 5

Hirshfeld surface of the title compound plotted over shape-index. 

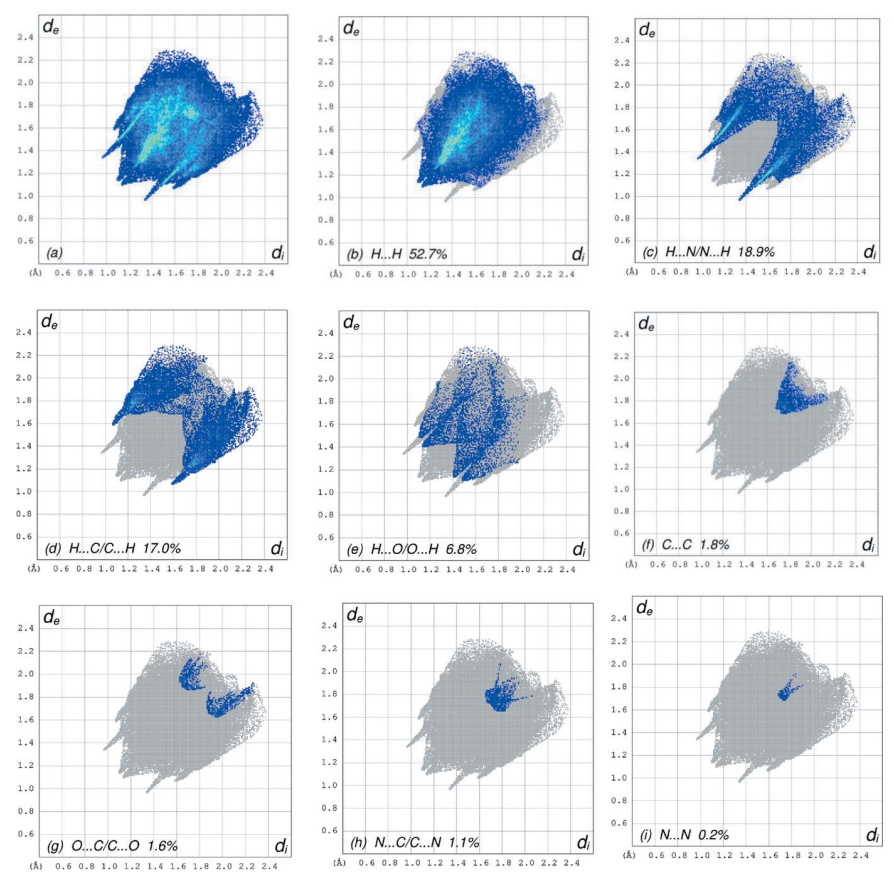

Figure 6

The full two-dimensional fingerprint plots for the title compound, showing $(a)$ all interactions, and delineated into $(b) \mathrm{H} \cdots \mathrm{H},(c) \mathrm{H} \cdots \mathrm{N} /$ $\mathrm{N} \cdots \mathrm{H},(d) \mathrm{H} \cdots \mathrm{C} / \mathrm{C} \cdots \mathrm{H},(e) \mathrm{H} \cdots \mathrm{O} / \mathrm{O} \cdots \mathrm{H},(f) \mathrm{C} \cdots \mathrm{C},(g) \mathrm{O} \cdots \mathrm{C} / \mathrm{C} \cdots \mathrm{O}$, (h) $\mathrm{N} \cdots \mathrm{C} / \mathrm{C} \cdots \mathrm{N}$ and $(i) \mathrm{N} \cdots \mathrm{N}$ interactions. The $d_{\mathrm{i}}$ and $d_{\mathrm{e}}$ values are the closest internal and external distances (in $\AA$ ) from given points on the Hirshfeld surface contacts.

symmetrical distribution of points, namely two pairs of thin and thick edges at $d_{\mathrm{e}}+d_{\mathrm{i}} \sim 2.53$ and $2.58 \AA$, respectively.
Table 2

Selected interatomic distances $(\AA)$.

\begin{tabular}{|c|c|c|c|}
\hline $\mathrm{O} 1 \cdots \mathrm{C} 11$ & 3.3134 (19) & $\mathrm{C} 2 \cdots \mathrm{C} 10^{\mathrm{iv}}$ & $3.586(2)$ \\
\hline $\mathrm{O} 1 \cdots \mathrm{C} 13^{\mathrm{i}}$ & $3.306(2)$ & $\mathrm{C} 2 \cdots \mathrm{C} 11$ & $3.559(2)$ \\
\hline $\mathrm{O} 1 \cdots \mathrm{C} 14^{\mathrm{i}}$ & $3.193(2)$ & $\mathrm{C} 2 \cdots \mathrm{C} 11^{\mathrm{vii}}$ & $3.589(2)$ \\
\hline $\mathrm{O} 1 \cdots \mathrm{H} 10 B$ & $2.341(17)$ & $\mathrm{C} 3 \cdots \mathrm{C} 10^{\mathrm{iv}}$ & $3.431(2)$ \\
\hline $\mathrm{O} 1 \cdots \mathrm{H} 9 B$ & $2.75(2)$ & $\mathrm{C} 4 \cdots \mathrm{C} 8^{\mathrm{iv}}$ & $3.502(2)$ \\
\hline $\mathrm{O} 1 \cdots \mathrm{H} 9 \mathrm{C}$ & $2.79(2)$ & $\mathrm{C} 5 \cdots \mathrm{C} 8^{\mathrm{iv}}$ & $3.491(2)$ \\
\hline $\mathrm{O} 1 \cdots \mathrm{H} 13 A^{\mathrm{ii}}$ & $2.696(17)$ & $\mathrm{C} 5 \cdots \mathrm{C}^{\mathrm{iv}}$ & $3.429(2)$ \\
\hline $\mathrm{O} 1 \cdots \mathrm{H} 13 B^{\mathrm{i}}$ & $2.62(2)$ & $\mathrm{C} 11 \cdots \mathrm{C} 13^{\mathrm{ii}}$ & $3.589(2)$ \\
\hline $\mathrm{O} 1 \cdots \mathrm{H} 14 A^{\mathrm{i}}$ & $2.752(18)$ & $\mathrm{C} 12 \cdots \mathrm{C} 13^{\mathrm{ii}}$ & $3.470(2)$ \\
\hline $\mathrm{N} 1 \cdots \mathrm{N} 2$ & $2.8013(17)$ & $\mathrm{C} 16 \cdots \mathrm{C} 16^{\mathrm{viii}}$ & $3.577(3)$ \\
\hline $\mathrm{N} 2 \cdots \mathrm{C}^{\mathrm{iii}}$ & $3.421(2)$ & $\mathrm{C} 2 \cdots \mathrm{H} 15 B^{\mathrm{vii}}$ & 2.991 (19) \\
\hline $\mathrm{N} 2 \cdots \mathrm{N} 5$ & 3.1514 (17) & $\mathrm{C} 2 \cdots \mathrm{H} 10 A$ & $2.600(17)$ \\
\hline $\mathrm{N} 4 \cdots \mathrm{C} 15$ & $3.364(2)$ & $\mathrm{C} 2 \cdots \mathrm{H} 10 B^{\mathrm{iv}}$ & $2.928(17)$ \\
\hline $\mathrm{N} 4 \cdots \mathrm{C} 12^{\mathrm{iv}}$ & $3.228(2)$ & $\mathrm{C} 8 \cdots \mathrm{H} 13 A^{\mathrm{ii}}$ & $2.918(18)$ \\
\hline $\mathrm{N} 5 \cdots \mathrm{C} 2$ & $3.343(2)$ & $\mathrm{C} 10 \cdots \mathrm{H} 2$ & $2.608(18)$ \\
\hline $\mathrm{N} 1 \cdots \mathrm{H} 5^{\mathrm{v}}$ & $2.680(18)$ & $\mathrm{C} 11 \cdots \mathrm{H} 2^{\mathrm{vii}}$ & $2.755(19)$ \\
\hline $\mathrm{N} 3 \cdots \mathrm{H} 15 B$ & $2.860(19)$ & $\mathrm{C} 12 \cdots \mathrm{H} 13 A^{\mathrm{ii}}$ & $2.973(17)$ \\
\hline $\mathrm{N} 4 \cdots \mathrm{H} 12^{\text {iv }}$ & $2.42(2)$ & $\mathrm{H} 2 \cdots \mathrm{H} 10 A$ & $2.07(2)$ \\
\hline $\mathrm{N} 4 \cdots \mathrm{H} 13 B^{\mathrm{ii}}$ & $2.944(19)$ & $\mathrm{H} 4 \cdots \mathrm{H} 16 B^{\mathrm{vi}}$ & $2.52(3)$ \\
\hline $\mathrm{N} 4 \cdots \mathrm{H} 15 B$ & $2.936(18)$ & $\mathrm{H} 9 B \cdots \mathrm{H} 15 A^{\mathrm{ii}}$ & $2.40(3)$ \\
\hline $\mathrm{N} 4 \cdots \mathrm{H} 3^{\mathrm{vi}}$ & $2.821(18)$ & $\mathrm{H} 13 A \cdots \mathrm{H} 15 A$ & $2.54(3)$ \\
\hline $\mathrm{N} 5 \cdots \mathrm{H} 2$ & $2.819(19)$ & $\mathrm{H} 14 A \cdots \mathrm{H} 16 A$ & $2.46(3)$ \\
\hline $\mathrm{N} 5 \cdots \mathrm{H} 10 B^{\text {iv }}$ & $2.733(17)$ & $\mathrm{H} 14 B \cdots \mathrm{H} 16 C$ & $2.57(3)$ \\
\hline $\mathrm{N} 5 \cdots \mathrm{H} 2^{\mathrm{vii}}$ & $2.932(18)$ & $\mathrm{H} 15 A \cdots \mathrm{H} 13 A$ & $2.54(3)$ \\
\hline $\mathrm{N} 5 \cdots \mathrm{H} 10 A^{\mathrm{vii}}$ & $2.676(18)$ & $\mathrm{H} 15 A \cdots \mathrm{H} 9 B^{\mathrm{ii}}$ & $2.40(3)$ \\
\hline $\mathrm{N} 5 \cdots \mathrm{H} 13 B^{\mathrm{ii}}$ & $2.93(2)$ & & \\
\hline
\end{tabular}

Symmetry codes: (i) $-x,-y,-z+1$; (ii) $-x+1,-y,-z+1$; (iii) $x-1, y, z$; (iv) $x+1, y, z ; \quad$ (v) $\quad-x+2,-y+1,-z+2 ; \quad$ (vi) $\quad-x+2,-y+1,-z+1$; $\quad$ (vii) $-x+1,-y+1,-z+1 ;($ viii) $-x,-y,-z$

The Hirshfeld surface representations with the function $d_{\text {norm }}$ plotted onto the surface are shown for the $\mathrm{H} \cdots \mathrm{H}$, $\mathrm{H} \cdots \mathrm{N} / \mathrm{N} \cdots \mathrm{H}, \mathrm{H} \cdots \mathrm{C} / \mathrm{C} \cdots \mathrm{H}$ and $\mathrm{H} \cdots \mathrm{O} / \mathrm{O} \cdots \mathrm{H}$ interactions in Fig. $7(a)-(d)$, respectively.

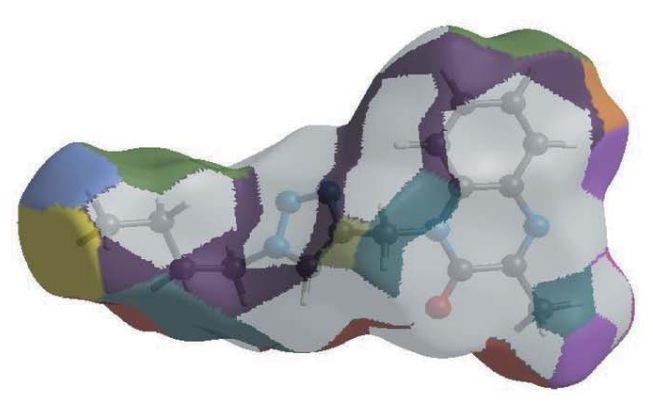

(a)

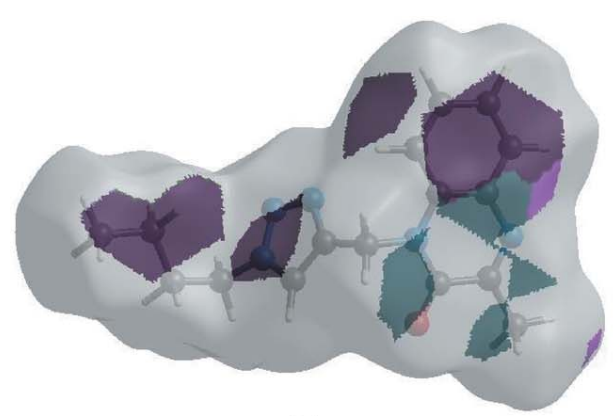

(c)

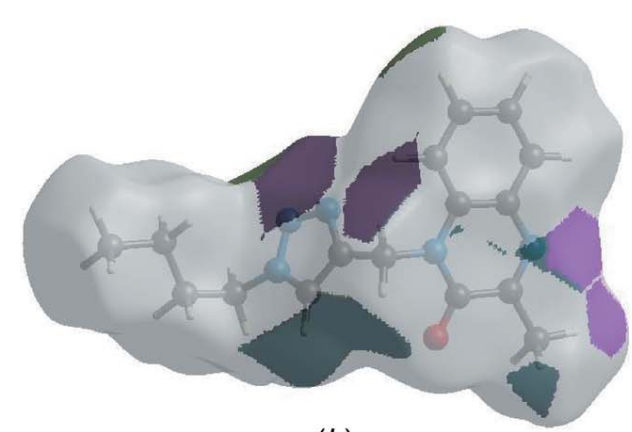

(b)

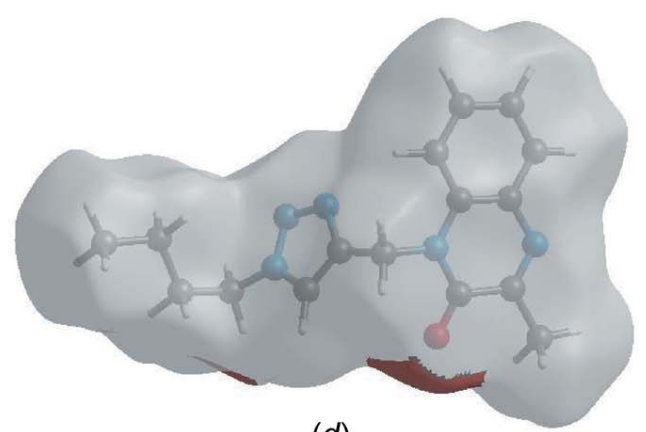

(d)

Figure 7

The Hirshfeld surface representations with the function $d_{\text {norm }}$ plotted onto the surface for $(a) \mathrm{H} \cdots \mathrm{H},(b) \mathrm{H} \cdots \mathrm{N} / \mathrm{N} \cdots \mathrm{H},(c) \mathrm{H} \cdots \mathrm{C} / \mathrm{C} \cdots \mathrm{H}$ and $(d) \mathrm{H} \cdots \mathrm{O} /$ $\mathrm{O} \cdots \mathrm{H}$ interactions. 
Table 3

Experimental details.

\begin{tabular}{|c|c|}
\hline \multicolumn{2}{|l|}{ Crystal data } \\
\hline Chemical formula & $\mathrm{C}_{16} \mathrm{H}_{19} \mathrm{~N}_{5} \mathrm{O}$ \\
\hline$M_{\mathrm{r}}$ & 297.36 \\
\hline Crystal system, space group & Triclinic, $P \overline{1}$ \\
\hline Temperature (K) & 150 \\
\hline$a, b, c(\AA)$ & 5.3265 (2), $9.9946(4), 14.5414(5)$ \\
\hline$\alpha, \beta, \gamma\left(^{\circ}\right)$ & $103.054(2), 100.039(2), 93.108(2)$ \\
\hline$V\left(\AA^{3}\right)$ & $739.03(5)$ \\
\hline$Z$ & 2 \\
\hline Radiation type & $\mathrm{Cu} K \alpha$ \\
\hline$\mu\left(\mathrm{mm}^{-1}\right)$ & 0.71 \\
\hline Crystal size $(\mathrm{mm})$ & $0.25 \times 0.21 \times 0.02$ \\
\hline \multicolumn{2}{|l|}{ Data collection } \\
\hline Diffractometer & $\begin{array}{l}\text { Bruker D8 VENTURE PHOTON } \\
100 \text { CMOS }\end{array}$ \\
\hline Absorption correction & $\begin{array}{l}\text { Multi-scan (SADABS; Krause et } \\
\quad \text { al., 2015) }\end{array}$ \\
\hline$T_{\min }, T_{\max }$ & $0.84,0.97$ \\
\hline $\begin{array}{l}\text { No. of measured, independent and } \\
\text { observed }[I>2 \sigma(I)] \text { reflections }\end{array}$ & $5735,2764,2281$ \\
\hline$R_{\text {int }}$ & 0.030 \\
\hline$(\sin \theta / \lambda)_{\max }\left(\AA^{-1}\right)$ & 0.617 \\
\hline \multicolumn{2}{|l|}{ Refinement } \\
\hline$R\left[F^{2}>2 \sigma\left(F^{2}\right)\right], w R\left(F^{2}\right), S$ & $0.041,0.100,1.07$ \\
\hline No. of reflections & 2764 \\
\hline No. of parameters & 276 \\
\hline $\mathrm{H}$-atom treatment & All $\mathrm{H}$-atom parameters refined \\
\hline$\Delta \rho_{\max }, \Delta \rho_{\min }\left(\mathrm{e} \AA^{-3}\right)$ & $0.25,-0.21$ \\
\hline
\end{tabular}

Computer programs: APEX3 and SAINT (Bruker, 2016), SHELXT (Sheldrick, 2015a), SHELXL2018 (Sheldrick, 2015b), DIAMOND (Brandenburg \& Putz, 2012) and SHELXTL (Sheldrick, 2008).

The Hirshfeld surface analysis confirms the importance of $\mathrm{H}$-atom contacts in establishing the packing. The large number of $\mathrm{H} \cdots \mathrm{H}, \mathrm{H} \cdots \mathrm{N} / \mathrm{N} \cdots \mathrm{H}, \mathrm{H} \cdots \mathrm{C} / \mathrm{C} \cdots \mathrm{H}$ and $\mathrm{H} \cdots \mathrm{O} / \mathrm{O} \cdots \mathrm{H}$ interactions suggests that van der Waals interactions and hydrogen bonding play the major roles in the crystal packing (Hathwar et al., 2015).

\section{Synthesis and crystallization}

To a solution of 3-methyl-1-(prop-2-ynyl)-3,4-dihydroquinoxalin-2 $(1 H)$-one $(0.68 \mathrm{mmol})$ in ethanol $(15 \mathrm{~mL})$ was added 1-azidobutane $(1.03 \mathrm{mmol})$. The reaction mixture was stirred under reflux for $72 \mathrm{~h}$. After completion of the reaction (monitored by TLC), the solution was concentrated and the residue was purified by column chromatography on silica gel by using as eluent the mixture (hexane/ethyl acetate 8:2). The solid product obtained was crystallized from ethanol to afford colourless crystals in $78 \%$ yield.

\section{Refinement}

Crystal data, data collection and structure refinement details are summarized in Table 3 . $\mathrm{H}$ atoms were located in a difference Fourier map and were freely refined.

\section{Funding information}

The support of NSF-MRI grant No. 1228232 for the purchase of the diffractometer and Tulane University for support of the
Tulane Crystallography Laboratory are gratefully acknowledged. $\mathrm{TH}$ is grateful to Hacettepe University Scientific Research Project Unit (grant No. 013 D04 602 004).

\section{References}

Abad, N., El Bakri, Y., Ramli, Y., Essassi, E. M. \& Mague, J. T. (2018b). IUCrData, 3, x180680.

Abad, N., El Bakri, Y., Sebhaoui, J., Ramli, Y., Essassi, E. M. \& Mague, J. T. (2018c). IUCrData, 3, x180519.

Abad, N., Ramli, Y., Sebbar, N. K., Kaur, M., Essassi, E. M. \& Jasinski, J. P. (2018a). IUCrData, 3, x180482.

Abbas, H. S., Al-Marhabi, A. R., Eissa, S. I. \& Ammar, Y. A. (2015). Bioorg. Med. Chem. 23, 6560-6572.

Attia, A. S., Abdel Aziz, A. A., Alfallous, K. A. \& El-Shahat, M. F. (2013). Polyhedron, 51, 243-254.

Benzeid, H., Ramli, Y., Vendier, L., Essassi, E. M. \& Ng, S. W. (2009). Acta Cryst. E65, o2196.

Benzeid, H., Vendier, L., Ramli, Y., Garrigues, B. \& Essassi, E. M. (2008). Acta Cryst. E64, o2234.

Brandenburg, K. \& Putz, H. (2012). DIAMOND. Crystal Impact GbR, Bonn, Germany.

Bruker (2016). APEX3, SADABS and SAINT. Bruker AXS Inc., Madison, Wisconsin, USA.

Caleb, A. A., Bouhfid, R., Essassi, E. M. \& El Ammari, L. (2009). Acta Cryst. E65, o2024-02025.

Ellouz, M., Sebbar, N. K., Essassi, E. M., Ouzidan, Y. \& Mague, J. T. (2015). Acta Cryst. E71, o1022-o1023.

Groom, C. R., Bruno, I. J., Lightfoot, M. P. \& Ward, S. C. (2016). Acta Cryst. B72, 171-179.

Guirado, A., López Sánchez, J. I., Ruiz-Alcaraz, A. J., Bautista, D. \& Gálvez, J. (2012). Eur. J. Med. Chem. 54, 87-94.

Hathwar, V. R., Sist, M., Jørgensen, M. R. V., Mamakhel, A. H., Wang, X., Hoffmann, C. M., Sugimoto, K., Overgaard, J. \& Iversen, B. B. (2015). IUCrJ, 2, 563-574.

Hirshfeld, H. L. (1977). Theor. Chim. Acta, 44, 129-138.

Jayatilaka, D., Grimwood, D. J., Lee, A., Lemay, A., Russel, A. J., Taylor, C., Wolff, S. K., Cassam-Chenai, P. \& Whitton, A. (2005). TONTO - A System for Computational Chemistry. Available at: http://hirshfeldsurface.net/

Krause, L., Herbst-Irmer, R., Sheldrick, G. M. \& Stalke, D. (2015). J. Appl. Cryst. 48, 3-10.

Kulkarni, N. V., Revankar, V. K., Kirasur, B. N. \& Hugar, M. H. (2012). Med. Chem. Res. 21, 663-671.

McKinnon, J. J., Jayatilaka, D. \& Spackman, M. A. (2007). Chem. Commun. pp. 3814-3816.

Ramli, Y., El Bakri, Y., 'El Ghayati, L., Essassi, E. M. \& Mague, J. T. (2018). IUCRData 3, x180390.

Ramli, Y. \& Essassi, E. M. (2015). Adv. Chem. Res. 27, 109-160.

Ramli, Y., Karrouchi, K., Essassi, E. M. \& El Ammari, L. (2013). Acta Cryst. E69, o1320-01321.

Ramli, Y., Missioui, M., El Fal, M., Ouhcine, M., Essassi, E. M. \& Mague, J. T. (2017). IUCrData, 2, x171424.

Ramli, Y., Moussaif, A., Zouihri, H., Lazar, S. \& Essassi, E. M. (2010a). Acta Cryst. E66, o1922.

Ramli, Y., Slimani, R., Zouihri, H., Lazar, S. \& Essassi, E. M. (2010b). Acta Cryst. E66, o1767.

Sebbar, N. K., Zerzouf, A., Essassi, E. M., Saadi, M. \& El Ammari, L. (2014). Acta Cryst. E70, o116.

Sheldrick, G. M. (2008). Acta Cryst. A64, 112-122.

Sheldrick, G. M. (2015a). Acta Cryst. A71, 3-8.

Sheldrick, G. M. (2015b). Acta Cryst. C71, 3-8.

Spackman, M. A. \& Jayatilaka, D. (2009). CrystEngComm, 11, 19-32.

Spackman, M. A., McKinnon, J. J. \& Jayatilaka, D. (2008). CrystEngComm, 10, 377-388.

Sridevi, K. B. C. H., Naidu, A. \& Sudhakaran, R. (2010). Eur. J. Chem. 7, 234-238. 
Teja, R., Kapu, S., Kadiyala, S., Dhanapal, V. \& Raman, A. N. (2016). J. Saudi Chem. Soc. 20, S387-S392.

Turner, M. J., McKinnon, J. J., Wolff, S. K., Grimwood, D. J., Spackman, P. R., Jayatilaka, D. \& Spackman, M. A. (2017). CrystalExplorer17. The University of Western Australia.
Venkatesan, P., Thamotharan, S., Ilangovan, A., Liang, H. \& Sundius, T. (2016). Spectrochim. Acta Part A, 153, 625-636.

Vieira, M., Pinheiro, C., Fernandes, R., Noronha, J. P. \& Prudêncio, C. (2014). Microbiol. Res. 169, 287-293. 


\section{supporting information}

Acta Cryst. (2018). E74, 1815-1820 [https://doi.org/10.1107/S205698901801589X]

Crystal structure and Hirshfeld surface analysis of 1-[(1-butyl-1H-1,2,3triazol-4-yl)methyl]-3-methylquinoxalin-2(1H)-one

Nadeem Abad, Youssef Ramli, Tuncer Hökelek, Nada Kheira Sebbar, Joel T. Mague and El Mokhtar Essassi

Computing details

Data collection: APEX3 (Bruker, 2016); cell refinement: SAINT (Bruker, 2016); data reduction: SAINT (Bruker, 2016); program(s) used to solve structure: SHELXT (Sheldrick, 2015a); program(s) used to refine structure: SHELXL2018 (Sheldrick, 2015b); molecular graphics: DIAMOND (Brandenburg \& Putz, 2012); software used to prepare material for publication: SHELXTL (Sheldrick, 2008).

\1-[(1-Butyl-1H-1,2,3-triazol-4-yl)methyl]-\3-methylquinoxalin-2(1H)-one

Crystal data

$\mathrm{C}_{16} \mathrm{H}_{19} \mathrm{~N}_{5} \mathrm{O}$

$M_{r}=297.36$

Triclinic, $P \overline{1}$

$a=5.3265(2) \AA$

$b=9.9946(4) \AA$

$c=14.5414(5) \AA$

$\alpha=103.054(2)^{\circ}$

$\beta=100.039(2)^{\circ}$

$\gamma=93.108(2)^{\circ}$

$V=739.03(5) \AA^{3}$

\section{Data collection}

Bruker D8 VENTURE PHOTON 100 CMOS diffractometer

Radiation source: INCOATEC I $\mu \mathrm{S}$ micro-focus source

Mirror monochromator

Detector resolution: 10.4167 pixels $\mathrm{mm}^{-1}$

$\omega$ scans

Absorption correction: multi-scan

(SADABS; Krause et al., 2015)

Refinement

Refinement on $F^{2}$

Least-squares matrix: full

$R\left[F^{2}>2 \sigma\left(F^{2}\right)\right]=0.041$

$w R\left(F^{2}\right)=0.100$

$S=1.07$

2764 reflections
$Z=2$

$F(000)=316$

$D_{\mathrm{x}}=1.336 \mathrm{Mg} \mathrm{m}^{-3}$

$\mathrm{Cu} K \alpha$ radiation, $\lambda=1.54178 \AA$

Cell parameters from 3995 reflections

$\theta=3.2-72.1^{\circ}$

$\mu=0.71 \mathrm{~mm}^{-1}$

$T=150 \mathrm{~K}$

Plate, colourless

$0.25 \times 0.21 \times 0.02 \mathrm{~mm}$

$T_{\min }=0.84, T_{\max }=0.97$

5735 measured reflections

2764 independent reflections

2281 reflections with $I>2 \sigma(I)$

$R_{\text {int }}=0.030$

$\theta_{\text {max }}=72.1^{\circ}, \theta_{\min }=3.2^{\circ}$

$h=-6 \rightarrow 6$

$k=-12 \rightarrow 11$

$l=-16 \rightarrow 17$

276 parameters

0 restraints

Primary atom site location: structure-invariant direct methods

Secondary atom site location: difference Fourier map 
Hydrogen site location: difference Fourier map All $\mathrm{H}$-atom parameters refined

$w=1 /\left[\sigma^{2}\left(F_{\mathrm{o}}^{2}\right)+(0.0403 P)^{2}+0.2108 P\right]$

where $P=\left(F_{\mathrm{o}}^{2}+2 F_{\mathrm{c}}^{2}\right) / 3$

$(\Delta / \sigma)_{\max }<0.001$
$\Delta \rho_{\max }=0.25$ e $\AA^{-3}$

$\Delta \rho_{\min }=-0.21$ e $\AA^{-3}$

Extinction correction: SHELXL2018 (Sheldrick, $2015 b), \mathrm{Fc}^{*}=\mathrm{kFc}\left[1+0.001 \mathrm{xFc}^{2} \lambda^{3} / \sin (2 \theta)\right]^{-1 / 4}$

Extinction coefficient: 0.0091 (10)

\section{Special details}

Geometry. All esds (except the esd in the dihedral angle between two 1.s. planes) are estimated using the full covariance matrix. The cell esds are taken into account individually in the estimation of esds in distances, angles and torsion angles; correlations between esds in cell parameters are only used when they are defined by crystal symmetry. An approximate (isotropic) treatment of cell esds is used for estimating esds involving l.s. planes.

Refinement. Refinement of $\mathrm{F}^{2}$ against ALL reflections. The weighted R-factor $\mathrm{wR}$ and goodness of fit $\mathrm{S}$ are based on $\mathrm{F}^{2}$, conventional R-factors $R$ are based on $F$, with $F$ set to zero for negative $F^{2}$. The threshold expression of $F^{2}>2 \operatorname{sigma}\left(F^{2}\right)$ is used only for calculating R-factors(gt) etc. and is not relevant to the choice of reflections for refinement. R-factors based on $\mathrm{F}^{2}$ are statistically about twice as large as those based on F, and R- factors based on ALL data will be even larger.

Fractional atomic coordinates and isotropic or equivalent isotropic displacement parameters $\left(\AA^{2}\right)$

\begin{tabular}{|c|c|c|c|c|}
\hline & $x$ & $y$ & $z$ & $U_{\text {iso }} * / U_{\text {eq }}$ \\
\hline $\mathrm{O} 1$ & $0.1373(2)$ & $0.25655(12)$ & $0.71106(8)$ & $0.0317(3)$ \\
\hline N1 & 0.6669 (2) & $0.43499(13)$ & $0.88772(9)$ & $0.0246(3)$ \\
\hline $\mathrm{N} 2$ & 0.4435 & $0.41368(12)$ & $0.69511(8)$ & $0.0214(3)$ \\
\hline N3 & $0.4218(2)$ & $0.10144(13)$ & $0.42094(8)$ & $0.0214(3)$ \\
\hline N4 & $0.6468(2)$ & $0.17916(13)$ & $0.43816(9)$ & $0.0257(3)$ \\
\hline N5 & $0.6307(2)$ & $0.29436(13)$ & $0.50153(9)$ & $0.0253(3)$ \\
\hline $\mathrm{C} 1$ & 0.6701 & $0.49930(15)$ & $0.73511(10)$ & $0.0214(3)$ \\
\hline $\mathrm{C} 2$ & 0.7938 & $0.57499(16)$ & $0.68289(11)$ & $0.0245(3)$ \\
\hline $\mathrm{H} 2$ & $0.722(3)$ & 0.5669 (19) & $0.6158(13)$ & $0.034(5)^{*}$ \\
\hline $\mathrm{C} 3$ & 1.0190 & $0.65725(16)$ & $0.72646(12)$ & $0.0282(4)$ \\
\hline $\mathrm{H} 3$ & $1.102(3)$ & 0.7133 (19) & $0.6888(13)$ & $0.032(5)^{*}$ \\
\hline $\mathrm{C} 4$ & $1.1277(3)$ & $0.66500(17)$ & $0.82210(12)$ & $0.0291(4)$ \\
\hline $\mathrm{H} 4$ & $1.280(4)$ & $0.720(2)$ & $0.8511(14)$ & $0.039(5)^{*}$ \\
\hline $\mathrm{C} 5$ & $1.0094(3)$ & $0.59047(16)$ & $0.87417(11)$ & $0.0267(3)$ \\
\hline $\mathrm{H} 5$ & $1.084(3)$ & $0.5905(18)$ & $0.9413(13)$ & $0.031(5)^{*}$ \\
\hline C6 & 0.7797 & $0.50745(15)$ & $0.83176(10)$ & $0.0227(3)$ \\
\hline $\mathrm{C} 7$ & 0.4566 & $0.35724(15)$ & $0.84935(10)$ & $0.0234(3)$ \\
\hline $\mathrm{C} 8$ & 0.3301 & $0.33717(15)$ & $0.74734(10)$ & $0.0231(3)$ \\
\hline $\mathrm{C} 9$ & 0.3266 & $0.28202(18)$ & $0.90846(12)$ & $0.0302(4)$ \\
\hline H9A & $0.422(4)$ & $0.301(2)$ & $0.9742(15)$ & $0.046(6)^{*}$ \\
\hline H9B & $0.310(4)$ & $0.182(2)$ & $0.8803(15)$ & $0.051(6)^{*}$ \\
\hline $\mathrm{H} 9 \mathrm{C}$ & $0.153(4)$ & $0.304(2)$ & 0.9088 (14) & $0.048(6)^{*}$ \\
\hline $\mathrm{C} 10$ & 0.3087 & $0.40222(16)$ & $0.59533(10)$ & $0.0232(3)$ \\
\hline $\mathrm{H} 10 \mathrm{~A}$ & $0.338(3)$ & $0.4926(18)$ & $0.5805(12)$ & $0.025(4)^{*}$ \\
\hline H10B & $0.125(3)$ & $0.3822(17)$ & $0.5929(12)$ & $0.028(4)^{*}$ \\
\hline $\mathrm{C} 11$ & 0.3937 & $0.28917(15)$ & $0.52445(10)$ & $0.0207(3)$ \\
\hline $\mathrm{C} 12$ & $0.2596(3)$ & $0.16668(16)$ & $0.47318(10)$ & $0.0222(3)$ \\
\hline H12 & $0.088(4)$ & 0.1281 (19) & $0.4678(13)$ & $0.033(5)^{*}$ \\
\hline C13 & 0.3800 & $-0.03351(16)$ & $0.35173(11)$ & $0.0265(3)$ \\
\hline $\mathrm{H} 13 \mathrm{~A}$ & $0.551(3)$ & $-0.0671(18)$ & $0.3519(12)$ & $0.030(4)^{*}$ \\
\hline
\end{tabular}




\begin{tabular}{lllll} 
H13B & $0.280(4)$ & $-0.096(2)$ & $0.3780(13)$ & $0.036(5)^{*}$ \\
C14 & $0.2469(3)$ & $-0.02638(17)$ & $0.25207(11)$ & $0.0262(3)$ \\
H14A & $0.210(3)$ & $-0.123(2)$ & $0.2122(13)$ & $0.034(5)^{*}$ \\
H14B & $0.078(3)$ & $0.0099(18)$ & $0.2559(12)$ & $0.031(5)^{*}$ \\
C15 & $0.3969(3)$ & $0.05954(18)$ & $0.20269(11)$ & $0.0293(4)$ \\
H15A & $0.570(4)$ & $0.0313(19)$ & $0.2062(13)$ & $0.037(5)^{*}$ \\
H15B & $0.412(3)$ & $0.160(2)$ & $0.2368(13)$ & $0.035(5)^{*}$ \\
C16 & $0.2667(4)$ & $0.0438(2)$ & $0.09843(13)$ & $0.0420(5)$ \\
H16A & $0.248(4)$ & $-0.057(2)$ & $0.0616(15)$ & $0.047(6)^{*}$ \\
H16B & $0.360(5)$ & $0.095(3)$ & $0.0645(17)$ & $0.067(7)^{*}$ \\
H16C & $0.092(5)$ & $0.071(3)$ & $0.0943(17)$ & $0.068(7)^{*}$ \\
\hline
\end{tabular}

Atomic displacement parameters $\left(\AA^{2}\right)$

\begin{tabular}{lllllll}
\hline & $U^{11}$ & $U^{22}$ & $U^{33}$ & $U^{12}$ & $U^{13}$ & $U^{23}$ \\
\hline O1 & $0.0292(6)$ & $0.0338(7)$ & $0.0292(6)$ & $-0.0076(5)$ & $0.0029(5)$ & $0.0062(5)$ \\
N1 & $0.0267(7)$ & $0.0249(7)$ & $0.0226(6)$ & $0.0033(5)$ & $0.0053(5)$ & $0.0059(5)$ \\
N2 & $0.0216(6)$ & $0.0233(7)$ & $0.0191(6)$ & $0.0024(5)$ & $0.0035(5)$ & $0.0048(5)$ \\
N3 & $0.0177(6)$ & $0.0237(7)$ & $0.0215(6)$ & $0.0004(5)$ & $0.0013(4)$ & $0.0051(5)$ \\
N4 & $0.0193(6)$ & $0.0290(7)$ & $0.0255(6)$ & $-0.0012(5)$ & $0.0031(5)$ & $0.0017(5)$ \\
N5 & $0.0212(6)$ & $0.0286(7)$ & $0.0246(6)$ & $-0.0006(5)$ & $0.0054(5)$ & $0.0030(5)$ \\
C1 & $0.0205(7)$ & $0.0208(7)$ & $0.0227(7)$ & $0.0034(5)$ & $0.0055(5)$ & $0.0032(6)$ \\
C2 & $0.0273(8)$ & $0.0238(8)$ & $0.0240(8)$ & $0.0050(6)$ & $0.0063(6)$ & $0.0071(6)$ \\
C3 & $0.0287(8)$ & $0.0261(8)$ & $0.0326(8)$ & $0.0015(6)$ & $0.0113(6)$ & $0.0090(7)$ \\
C4 & $0.0234(8)$ & $0.0284(8)$ & $0.0330(8)$ & $-0.0026(6)$ & $0.0049(6)$ & $0.0038(7)$ \\
C5 & $0.0252(8)$ & $0.0291(8)$ & $0.0234(8)$ & $0.0019(6)$ & $0.0026(6)$ & $0.0029(6)$ \\
C6 & $0.0232(7)$ & $0.0224(8)$ & $0.0229(7)$ & $0.0030(6)$ & $0.0062(6)$ & $0.0046(6)$ \\
C7 & $0.0265(8)$ & $0.0209(8)$ & $0.0236(7)$ & $0.0042(6)$ & $0.0067(6)$ & $0.0054(6)$ \\
C8 & $0.0235(7)$ & $0.0227(8)$ & $0.0239(7)$ & $0.0033(6)$ & $0.0068(6)$ & $0.0052(6)$ \\
C9 & $0.0348(9)$ & $0.0297(9)$ & $0.0268(8)$ & $-0.0017(7)$ & $0.0071(7)$ & $0.0084(7)$ \\
C10 & $0.0219(7)$ & $0.0264(8)$ & $0.0208(7)$ & $0.0037(6)$ & $0.0021(6)$ & $0.0057(6)$ \\
C11 & $0.0182(7)$ & $0.0260(8)$ & $0.0194(7)$ & $0.0025(5)$ & $0.0027(5)$ & $0.0091(6)$ \\
C12 & $0.0164(7)$ & $0.0282(8)$ & $0.0226(7)$ & $0.0004(6)$ & $0.0037(5)$ & $0.0077(6)$ \\
C13 & $0.0294(8)$ & $0.0205(8)$ & $0.0269(8)$ & $0.0011(6)$ & $0.0024(6)$ & $0.0029(6)$ \\
C14 & $0.0235(8)$ & $0.0261(8)$ & $0.0251(8)$ & $-0.0013(6)$ & $0.0007(6)$ & $0.0021(6)$ \\
C15 & $0.0307(9)$ & $0.0296(9)$ & $0.0263(8)$ & $-0.0005(7)$ & $0.0050(6)$ & $0.0049(7)$ \\
C16 & $0.0528(12)$ & $0.0436(12)$ & $0.0286(9)$ & $-0.0001(9)$ & $0.0044(8)$ & $0.0101(8)$ \\
& & & & & & \\
\hline & & & & & &
\end{tabular}

Geometric parameters ( $\left.\AA,{ }^{\circ}\right)$

\begin{tabular}{llll}
\hline $\mathrm{O} 1-\mathrm{C} 8$ & $1.2292(18)$ & $\mathrm{C} 7-\mathrm{C} 9$ & $1.492(2)$ \\
$\mathrm{N} 1-\mathrm{C} 7$ & $1.2891(19)$ & $\mathrm{C} 9-\mathrm{H} 9 \mathrm{~A}$ & $0.97(2)$ \\
$\mathrm{N} 1-\mathrm{C} 6$ & $1.3917(19)$ & $\mathrm{C} 9-\mathrm{H} 9 \mathrm{~B}$ & $0.98(2)$ \\
$\mathrm{N} 2-\mathrm{C} 8$ & $1.3780(19)$ & $\mathrm{C} 9-\mathrm{H} 9 \mathrm{C}$ & $0.96(2)$ \\
$\mathrm{N} 2-\mathrm{C} 1$ & $1.3951(18)$ & $\mathrm{C} 10-\mathrm{C} 11$ & $1.495(2)$ \\
$\mathrm{N} 2-\mathrm{C} 10$ & $1.4787(17)$ & $\mathrm{C} 10-\mathrm{H} 10 \mathrm{~A}$ & $0.986(18)$ \\
$\mathrm{N} 3-\mathrm{N} 4$ & $1.3451(17)$ & $\mathrm{C} 10-\mathrm{H} 10 \mathrm{~B}$ & $0.982(18)$ \\
$\mathrm{N} 3-\mathrm{C} 12$ & $1.3471(18)$ & $\mathrm{C} 11-\mathrm{C} 12$ & $1.368(2)$
\end{tabular}




\begin{tabular}{|c|c|c|c|}
\hline N3-C13 & $1.4690(19)$ & $\mathrm{C} 12-\mathrm{H} 12$ & $0.954(19)$ \\
\hline N4-N5 & $1.3196(18)$ & $\mathrm{C} 13-\mathrm{C} 14$ & $1.517(2)$ \\
\hline $\mathrm{N} 5-\mathrm{C} 11$ & $1.3617(18)$ & $\mathrm{C} 13-\mathrm{H} 13 \mathrm{~A}$ & $0.986(18)$ \\
\hline $\mathrm{C} 1-\mathrm{C} 2$ & $1.401(2)$ & C13-H13B & $0.98(2)$ \\
\hline $\mathrm{C} 1-\mathrm{C} 6$ & $1.407(2)$ & $\mathrm{C} 14-\mathrm{C} 15$ & $1.515(2)$ \\
\hline $\mathrm{C} 2-\mathrm{C} 3$ & $1.382(2)$ & $\mathrm{C} 14-\mathrm{H} 14 \mathrm{~A}$ & $0.996(19)$ \\
\hline $\mathrm{C} 2-\mathrm{H} 2$ & $0.966(18)$ & C14-H14B & $0.992(18)$ \\
\hline $\mathrm{C} 3-\mathrm{C} 4$ & $1.393(2)$ & $\mathrm{C} 15-\mathrm{C} 16$ & $1.522(2)$ \\
\hline $\mathrm{C} 3-\mathrm{H} 3$ & 1.003 (19) & C15-H15A & $0.977(19)$ \\
\hline $\mathrm{C} 4-\mathrm{C} 5$ & $1.377(2)$ & C15-H15B & $1.005(19)$ \\
\hline $\mathrm{C} 4-\mathrm{H} 4$ & $0.93(2)$ & C16-H16A & $1.02(2)$ \\
\hline $\mathrm{C} 5-\mathrm{C} 6$ & $1.401(2)$ & C16-H16B & $0.96(3)$ \\
\hline $\mathrm{C} 5-\mathrm{H} 5$ & $0.988(18)$ & $\mathrm{C} 16-\mathrm{H} 16 \mathrm{C}$ & $0.98(3)$ \\
\hline $\mathrm{C} 7-\mathrm{C} 8$ & $1.482(2)$ & & \\
\hline $\mathrm{O} 1 \cdots \mathrm{C} 11$ & 3.3134 (19) & $\mathrm{C} 4 \cdots \mathrm{C} 8^{\mathrm{iv}}$ & $3.502(2)$ \\
\hline $\mathrm{O} 1 \cdots \mathrm{C} 13^{\mathrm{i}}$ & $3.306(2)$ & $\mathrm{C} 5 \cdots \mathrm{C} 8^{\mathrm{iv}}$ & $3.491(2)$ \\
\hline $\mathrm{O} 1 \cdots \mathrm{C} 14^{\mathrm{i}}$ & $3.193(2)$ & $\mathrm{C} 5 \cdots \mathrm{C} 7^{\text {iv }}$ & $3.429(2)$ \\
\hline $\mathrm{O} 1 \cdots \mathrm{H} 10 \mathrm{~B}$ & $2.341(17)$ & $\mathrm{C} 11 \cdots \mathrm{C} 13^{\mathrm{ii}}$ & $3.589(2)$ \\
\hline O1 $\cdots$ H9B & $2.75(2)$ & $\mathrm{C} 12 \cdots \mathrm{C} 13^{\mathrm{ii}}$ & $3.470(2)$ \\
\hline $\mathrm{O} 1 \cdots \mathrm{H} 9 \mathrm{C}$ & $2.79(2)$ & $\mathrm{C} 16 \cdots \mathrm{C} 16^{\text {viii }}$ & $3.577(3)$ \\
\hline $\mathrm{O} 1 \cdots \mathrm{H} 13 \mathrm{~A}^{\mathrm{ii}}$ & $2.696(17)$ & $\mathrm{C} 2 \cdots \mathrm{H} 15 \mathrm{~B}^{\mathrm{vii}}$ & $2.991(19)$ \\
\hline $\mathrm{O} 1 \cdots \mathrm{H} 13 \mathrm{~B}^{\mathrm{i}}$ & $2.62(2)$ & $\mathrm{C} 2 \cdots \mathrm{H} 10 \mathrm{~A}$ & $2.600(17)$ \\
\hline $\mathrm{O} 1 \cdots \mathrm{H} 14 \mathrm{~A}^{\mathrm{i}}$ & $2.752(18)$ & $\mathrm{C} 2 \cdots \mathrm{H} 10 \mathrm{~B}^{\mathrm{iv}}$ & $2.928(17)$ \\
\hline $\mathrm{N} 1 \cdots \mathrm{N} 2$ & $2.8013(17)$ & $\mathrm{C} 3 \cdots \mathrm{H} 15 \mathrm{~B}^{\mathrm{vii}}$ & $3.055(18)$ \\
\hline $\mathrm{N} 2 \cdots \mathrm{C} 3^{\mathrm{iii}}$ & $3.421(2)$ & $\mathrm{C} 4 \cdots \mathrm{H} 16 \mathrm{C}^{\mathrm{vii}}$ & $3.03(3)$ \\
\hline $\mathrm{N} 2 \cdots \mathrm{N} 5$ & $3.1514(17)$ & $\mathrm{C} 7 \cdots \mathrm{H} 14 \mathrm{~A}^{\mathrm{ii}}$ & $3.081(19)$ \\
\hline $\mathrm{N} 4 \cdots \mathrm{C} 15$ & $3.364(2)$ & $\mathrm{C} 8 \cdots \mathrm{H} 13 \mathrm{~A}^{\mathrm{ii}}$ & $2.918(18)$ \\
\hline $\mathrm{N} 4 \cdots \mathrm{C} 12^{\mathrm{iv}}$ & $3.228(2)$ & $\mathrm{C} 10 \cdots \mathrm{H} 2$ & $2.608(18)$ \\
\hline $\mathrm{N} 5 \cdots \mathrm{C} 2$ & $3.343(2)$ & $\mathrm{C} 11 \cdots \mathrm{H} 2^{\mathrm{vii}}$ & $2.755(19)$ \\
\hline $\mathrm{N} 1 \cdots \mathrm{H} 5^{\mathrm{v}}$ & $2.680(18)$ & $\mathrm{C} 11 \cdots \mathrm{H} 13 \mathrm{~B}^{\mathrm{ii}}$ & $3.08(2)$ \\
\hline $\mathrm{N} 3 \cdots \mathrm{H} 15 \mathrm{~B}$ & 2.860 (19) & $\mathrm{C} 11 \cdots \mathrm{H} 2$ & $3.086(19)$ \\
\hline $\mathrm{N} 4 \cdots \mathrm{H} 12^{\mathrm{iv}}$ & $2.42(2)$ & $\mathrm{C} 12 \cdots \mathrm{H} 13 \mathrm{~A}^{\mathrm{ii}}$ & $2.973(17)$ \\
\hline $\mathrm{N} 4 \cdots \mathrm{H} 13 \mathrm{~B}^{\mathrm{ii}}$ & $2.944(19)$ & $\mathrm{C} 15 \cdots \mathrm{H} 4^{\mathrm{vi}}$ & $3.04(2)$ \\
\hline $\mathrm{N} 4 \cdots \mathrm{H} 15 \mathrm{~B}$ & $2.936(18)$ & $\mathrm{C} 15 \cdots \mathrm{H} 9 \mathrm{~B}^{\mathrm{ii}}$ & $3.08(2)$ \\
\hline $\mathrm{N} 4 \cdots \mathrm{H}^{\mathrm{vi}}$ & $2.821(18)$ & $\mathrm{C} 16 \cdots \mathrm{H} 16 \mathrm{C}^{\mathrm{viii}}$ & $3.04(2)$ \\
\hline $\mathrm{N} 5 \cdots \mathrm{H} 2$ & 2.819 (19) & $\mathrm{H} 2 \cdots \mathrm{H} 10 \mathrm{~A}$ & $2.07(2)$ \\
\hline $\mathrm{N} 5 \cdots \mathrm{H} 10 \mathrm{~B}^{\mathrm{iv}}$ & $2.733(17)$ & $\mathrm{H} 4 \cdots \mathrm{H} 16 \mathrm{~B}^{\mathrm{vi}}$ & $2.52(3)$ \\
\hline $\mathrm{N} 5 \cdots \mathrm{H} 2^{\text {vii }}$ & $2.932(18)$ & H9B $\cdots H 15 A^{\mathrm{ii}}$ & $2.40(3)$ \\
\hline $\mathrm{N} 5 \cdots \mathrm{H} 10 \mathrm{~A}^{\mathrm{vii}}$ & $2.676(18)$ & H13A $\cdots H 15 A$ & $2.54(3)$ \\
\hline $\mathrm{N} 5 \cdots \mathrm{H} 13 \mathrm{~B}^{\mathrm{ii}}$ & $2.93(2)$ & $\mathrm{H} 14 \mathrm{~A} \cdots \mathrm{H} 16 \mathrm{~A}$ & $2.46(3)$ \\
\hline $\mathrm{C} 2 \cdots \mathrm{C} 10^{\mathrm{iv}}$ & $3.586(2)$ & H14B $\cdots \mathrm{H} 16 \mathrm{C}$ & $2.57(3)$ \\
\hline $\mathrm{C} 2 \cdots \mathrm{C} 11$ & $3.559(2)$ & $\mathrm{H} 15 \mathrm{~A} \cdots \mathrm{H} 13 \mathrm{~A}$ & $2.54(3)$ \\
\hline $\mathrm{C} 2 \cdots \mathrm{C} 11^{\mathrm{vii}}$ & $3.589(2)$ & $\mathrm{H} 15 \mathrm{~A} \cdots \mathrm{H} \mathrm{B}^{\mathrm{ii}}$ & $2.40(3)$ \\
\hline $\mathrm{C} 3 \cdots \mathrm{C} 10^{\mathrm{iv}}$ & $3.431(2)$ & & \\
\hline $\mathrm{C} 7-\mathrm{N} 1-\mathrm{C} 6$ & $118.81(13)$ & $\mathrm{H} 9 \mathrm{~B}-\mathrm{C} 9-\mathrm{H} 9 \mathrm{C}$ & $104.4(17)$ \\
\hline $\mathrm{C} 8-\mathrm{N} 2-\mathrm{C} 1$ & $121.65(12)$ & $\mathrm{N} 2-\mathrm{C} 10-\mathrm{C} 11$ & $112.53(12)$ \\
\hline
\end{tabular}




\begin{tabular}{|c|c|}
\hline $\mathrm{C} 8-\mathrm{N} 2-\mathrm{C} 10$ & $116.29(12)$ \\
\hline $\mathrm{C} 1-\mathrm{N} 2-\mathrm{C} 10$ & $122.04(12)$ \\
\hline $\mathrm{N} 4-\mathrm{N} 3-\mathrm{C} 12$ & $110.55(12)$ \\
\hline $\mathrm{N} 4-\mathrm{N} 3-\mathrm{C} 13$ & $120.31(12)$ \\
\hline $\mathrm{C} 12-\mathrm{N} 3-\mathrm{C} 13$ & $129.14(13)$ \\
\hline $\mathrm{N} 5-\mathrm{N} 4-\mathrm{N} 3$ & $107.55(11)$ \\
\hline $\mathrm{N} 4-\mathrm{N} 5-\mathrm{C} 11$ & $108.38(12)$ \\
\hline $\mathrm{N} 2-\mathrm{C} 1-\mathrm{C} 2$ & $122.89(13)$ \\
\hline $\mathrm{N} 2-\mathrm{C} 1-\mathrm{C} 6$ & $118.01(13)$ \\
\hline $\mathrm{C} 2-\mathrm{C} 1-\mathrm{C} 6$ & $119.10(14)$ \\
\hline $\mathrm{C} 3-\mathrm{C} 2-\mathrm{C} 1$ & $120.11(14)$ \\
\hline $\mathrm{C} 3-\mathrm{C} 2-\mathrm{H} 2$ & $120.6(11)$ \\
\hline $\mathrm{C} 1-\mathrm{C} 2-\mathrm{H} 2$ & $119.3(11)$ \\
\hline $\mathrm{C} 2-\mathrm{C} 3-\mathrm{C} 4$ & $120.75(15)$ \\
\hline $\mathrm{C} 2-\mathrm{C} 3-\mathrm{H} 3$ & $119.2(10)$ \\
\hline $\mathrm{C} 4-\mathrm{C} 3-\mathrm{H} 3$ & $120.0(10)$ \\
\hline $\mathrm{C} 5-\mathrm{C} 4-\mathrm{C} 3$ & $119.81(15)$ \\
\hline $\mathrm{C} 5-\mathrm{C} 4-\mathrm{H} 4$ & $119.6(12)$ \\
\hline $\mathrm{C} 3-\mathrm{C} 4-\mathrm{H} 4$ & $120.6(12)$ \\
\hline $\mathrm{C} 4-\mathrm{C} 5-\mathrm{C} 6$ & $120.42(14)$ \\
\hline $\mathrm{C} 4-\mathrm{C} 5-\mathrm{H} 5$ & $122.2(11)$ \\
\hline $\mathrm{C} 6-\mathrm{C} 5-\mathrm{H} 5$ & $117.3(11)$ \\
\hline $\mathrm{N} 1-\mathrm{C} 6-\mathrm{C} 5$ & $118.15(13)$ \\
\hline $\mathrm{N} 1-\mathrm{C} 6-\mathrm{C} 1$ & $122.05(13)$ \\
\hline $\mathrm{C} 5-\mathrm{C} 6-\mathrm{C} 1$ & $119.80(14)$ \\
\hline $\mathrm{N} 1-\mathrm{C} 7-\mathrm{C} 8$ & $123.58(14)$ \\
\hline $\mathrm{N} 1-\mathrm{C} 7-\mathrm{C} 9$ & $120.01(13)$ \\
\hline $\mathrm{C} 8-\mathrm{C} 7-\mathrm{C} 9$ & $116.41(13)$ \\
\hline $\mathrm{O} 1-\mathrm{C} 8-\mathrm{N} 2$ & $121.86(13)$ \\
\hline $\mathrm{O} 1-\mathrm{C} 8-\mathrm{C} 7$ & $122.32(14)$ \\
\hline $\mathrm{N} 2-\mathrm{C} 8-\mathrm{C} 7$ & $115.82(13)$ \\
\hline $\mathrm{C} 7-\mathrm{C} 9-\mathrm{H} 9 \mathrm{~A}$ & $111.1(12)$ \\
\hline $\mathrm{C} 7-\mathrm{C} 9-\mathrm{H} 9 \mathrm{~B}$ & $110.3(12)$ \\
\hline $\mathrm{H} 9 \mathrm{~A}-\mathrm{C} 9-\mathrm{H} 9 \mathrm{~B}$ & $109.1(17)$ \\
\hline $\mathrm{C} 7-\mathrm{C} 9-\mathrm{H} 9 \mathrm{C}$ & $112.1(12)$ \\
\hline $\mathrm{H} 9 \mathrm{~A}-\mathrm{C} 9-\mathrm{H} 9 \mathrm{C}$ & $109.6(16)$ \\
\hline $\mathrm{C} 12-\mathrm{N} 3-\mathrm{N} 4-\mathrm{N} 5$ & $0.11(16)$ \\
\hline $\mathrm{C} 13-\mathrm{N} 3-\mathrm{N} 4-\mathrm{N} 5$ & $179.17(12)$ \\
\hline $\mathrm{N} 3-\mathrm{N} 4-\mathrm{N} 5-\mathrm{C} 11$ & $0.03(15)$ \\
\hline $\mathrm{C} 8-\mathrm{N} 2-\mathrm{C} 1-\mathrm{C} 2$ & $178.01(13)$ \\
\hline $\mathrm{C} 10-\mathrm{N} 2-\mathrm{C} 1-\mathrm{C} 2$ & $-3.4(2)$ \\
\hline $\mathrm{C} 8-\mathrm{N} 2-\mathrm{C} 1-\mathrm{C} 6$ & $-1.0(2)$ \\
\hline $\mathrm{C} 10-\mathrm{N} 2-\mathrm{C} 1-\mathrm{C} 6$ & $177.67(13)$ \\
\hline $\mathrm{N} 2-\mathrm{C} 1-\mathrm{C} 2-\mathrm{C} 3$ & $-179.52(13)$ \\
\hline $\mathrm{C} 6-\mathrm{C} 1-\mathrm{C} 2-\mathrm{C} 3$ & $-0.6(2)$ \\
\hline $\mathrm{C} 1-\mathrm{C} 2-\mathrm{C} 3-\mathrm{C} 4$ & $0.7(2)$ \\
\hline $\mathrm{C} 2$ & \\
\hline
\end{tabular}

\begin{tabular}{|c|c|}
\hline $\mathrm{N} 2-\mathrm{C} 10-\mathrm{H} 10 \mathrm{~A}$ & $107.5(10)$ \\
\hline $\mathrm{C} 11-\mathrm{C} 10-\mathrm{H} 10 \mathrm{~A}$ & $111.5(10)$ \\
\hline $\mathrm{N} 2-\mathrm{C} 10-\mathrm{H} 10 \mathrm{~B}$ & $107.9(10)$ \\
\hline $\mathrm{C} 11-\mathrm{C} 10-\mathrm{H} 10 \mathrm{~B}$ & $108.4(10)$ \\
\hline $\mathrm{H} 10 \mathrm{~A}-\mathrm{C} 10-\mathrm{H} 10 \mathrm{~B}$ & $108.9(14)$ \\
\hline $\mathrm{N} 5-\mathrm{C} 11-\mathrm{C} 12$ & $108.52(13)$ \\
\hline $\mathrm{N} 5-\mathrm{C} 11-\mathrm{C} 10$ & $123.12(13)$ \\
\hline $\mathrm{C} 12-\mathrm{C} 11-\mathrm{C} 10$ & $128.37(13)$ \\
\hline $\mathrm{N} 3-\mathrm{C} 12-\mathrm{C} 11$ & $105.01(13)$ \\
\hline $\mathrm{N} 3-\mathrm{C} 12-\mathrm{H} 12$ & $121.9(11)$ \\
\hline $\mathrm{C} 11-\mathrm{C} 12-\mathrm{H} 12$ & $133.1(11)$ \\
\hline $\mathrm{N} 3-\mathrm{C} 13-\mathrm{C} 14$ & $112.79(13)$ \\
\hline $\mathrm{N} 3-\mathrm{C} 13-\mathrm{H} 13 \mathrm{~A}$ & $105.8(10)$ \\
\hline $\mathrm{C} 14-\mathrm{C} 13-\mathrm{H} 13 \mathrm{~A}$ & $112.3(10)$ \\
\hline $\mathrm{N} 3-\mathrm{C} 13-\mathrm{H} 13 \mathrm{~B}$ & $107.0(11)$ \\
\hline $\mathrm{C} 14-\mathrm{C} 13-\mathrm{H} 13 \mathrm{~B}$ & $111.2(11)$ \\
\hline $\mathrm{H} 13 \mathrm{~A}-\mathrm{C} 13-\mathrm{H} 13 \mathrm{~B}$ & $107.3(15)$ \\
\hline $\mathrm{C} 15-\mathrm{C} 14-\mathrm{C} 13$ & $114.99(13)$ \\
\hline $\mathrm{C} 15-\mathrm{C} 14-\mathrm{H} 14 \mathrm{~A}$ & $109.5(10)$ \\
\hline $\mathrm{C} 13-\mathrm{C} 14-\mathrm{H} 14 \mathrm{~A}$ & $107.1(10)$ \\
\hline $\mathrm{C} 15-\mathrm{C} 14-\mathrm{H} 14 \mathrm{~B}$ & $109.1(10)$ \\
\hline $\mathrm{C} 13-\mathrm{C} 14-\mathrm{H} 14 \mathrm{~B}$ & $109.3(10)$ \\
\hline $\mathrm{H} 14 \mathrm{~A}-\mathrm{C} 14-\mathrm{H} 14 \mathrm{~B}$ & $106.5(14)$ \\
\hline $\mathrm{C} 14-\mathrm{C} 15-\mathrm{C} 16$ & $111.11(14)$ \\
\hline $\mathrm{C} 14-\mathrm{C} 15-\mathrm{H} 15 \mathrm{~A}$ & $109.0(11)$ \\
\hline $\mathrm{C} 16-\mathrm{C} 15-\mathrm{H} 15 \mathrm{~A}$ & $110.5(11)$ \\
\hline $\mathrm{C} 14-\mathrm{C} 15-\mathrm{H} 15 \mathrm{~B}$ & $110.4(10)$ \\
\hline $\mathrm{C} 16-\mathrm{C} 15-\mathrm{H} 15 \mathrm{~B}$ & $108.5(10)$ \\
\hline $\mathrm{H} 15 \mathrm{~A}-\mathrm{C} 15-\mathrm{H} 15 \mathrm{~B}$ & $107.3(15)$ \\
\hline $\mathrm{C} 15-\mathrm{C} 16-\mathrm{H} 16 \mathrm{~A}$ & $110.7(12)$ \\
\hline $\mathrm{C} 15-\mathrm{C} 16-\mathrm{H} 16 \mathrm{~B}$ & $113.1(14)$ \\
\hline $\mathrm{H} 16 \mathrm{~A}-\mathrm{C} 16-\mathrm{H} 16 \mathrm{~B}$ & $106.6(17)$ \\
\hline $\mathrm{C} 15-\mathrm{C} 16-\mathrm{H} 16 \mathrm{C}$ & $111.0(14)$ \\
\hline $\mathrm{H} 16 \mathrm{~A}-\mathrm{C} 16-\mathrm{H} 16 \mathrm{C}$ & $105.7(18)$ \\
\hline $\mathrm{H} 16 \mathrm{~B}-\mathrm{C} 16-\mathrm{H} 16 \mathrm{C}$ & $109(2)$ \\
\hline $\mathrm{C} 1-\mathrm{N} 2-\mathrm{C} 8-\mathrm{O} 1$ & $-176.40(13)$ \\
\hline $\mathrm{C} 10-\mathrm{N} 2-\mathrm{C} 8-\mathrm{O} 1$ & $4.9(2)$ \\
\hline $\mathrm{C} 1-\mathrm{N} 2-\mathrm{C} 8-\mathrm{C} 7$ & $2.99(19)$ \\
\hline $\mathrm{C} 10-\mathrm{N} 2-\mathrm{C} 8-\mathrm{C} 7$ & $-175.71(12)$ \\
\hline $\mathrm{N} 1-\mathrm{C} 7-\mathrm{C} 8-\mathrm{O} 1$ & $175.85(14)$ \\
\hline $\mathrm{C} 9-\mathrm{C} 7-\mathrm{C} 8-\mathrm{O} 1$ & $-4.4(2)$ \\
\hline $\mathrm{N} 1-\mathrm{C} 7-\mathrm{C} 8-\mathrm{N} 2$ & $-3.5(2)$ \\
\hline $\mathrm{C} 9-\mathrm{C} 7-\mathrm{C} 8-\mathrm{N} 2$ & $176.18(13)$ \\
\hline $\mathrm{C} 8-\mathrm{N} 2-\mathrm{C} 10-\mathrm{C} 11$ & $-90.45(15)$ \\
\hline $\mathrm{C} 1-\mathrm{N} 2-\mathrm{C} 10-\mathrm{C} 11$ & $90.85(16)$ \\
\hline $\mathrm{N} 4-\mathrm{N} 5-\mathrm{C} 11-\mathrm{C} 12$ & $-0.15(16)$ \\
\hline
\end{tabular}




\begin{tabular}{llll}
$\mathrm{C} 3-\mathrm{C} 4-\mathrm{C} 5-\mathrm{C} 6$ & $-0.3(2)$ & $\mathrm{N} 4-\mathrm{N} 5-\mathrm{C} 11-\mathrm{C} 10$ & $179.35(13)$ \\
$\mathrm{C} 7-\mathrm{N} 1-\mathrm{C} 6-\mathrm{C} 5$ & $-179.41(14)$ & $\mathrm{N} 2-\mathrm{C} 10-\mathrm{C} 11-\mathrm{N} 5$ & $-69.14(18)$ \\
$\mathrm{C} 7-\mathrm{N} 1-\mathrm{C} 6-\mathrm{C} 1$ & $0.5(2)$ & $\mathrm{N} 2-\mathrm{C} 10-\mathrm{C} 11-\mathrm{C} 12$ & $110.25(16)$ \\
$\mathrm{C} 4-\mathrm{C} 5-\mathrm{C} 6-\mathrm{N} 1$ & $-179.55(14)$ & $\mathrm{N} 4-\mathrm{N} 3-\mathrm{C} 12-\mathrm{C} 11$ & $-0.20(15)$ \\
$\mathrm{C} 4-\mathrm{C} 5-\mathrm{C} 6-\mathrm{C} 1$ & $0.5(2)$ & $\mathrm{C} 13-\mathrm{N} 3-\mathrm{C} 12-\mathrm{C} 11$ & $-179.15(13)$ \\
$\mathrm{N} 2-\mathrm{C} 1-\mathrm{C} 6-\mathrm{N} 1$ & $-1.0(2)$ & $\mathrm{N} 5-\mathrm{C} 11-\mathrm{C} 12-\mathrm{N} 3$ & $0.22(16)$ \\
$\mathrm{C} 2-\mathrm{C} 1-\mathrm{C} 6-\mathrm{N} 1$ & $180.00(13)$ & $\mathrm{C} 10-\mathrm{C} 11-\mathrm{C} 12-\mathrm{N} 3$ & $-179.25(13)$ \\
$\mathrm{N} 2-\mathrm{C} 1-\mathrm{C} 6-\mathrm{C} 5$ & $178.96(13)$ & $\mathrm{N} 4-\mathrm{N} 3-\mathrm{C} 13-\mathrm{C} 14$ & $-95.26(16)$ \\
$\mathrm{C} 2-\mathrm{C} 1-\mathrm{C} 6-\mathrm{C} 5$ & $0.0(2)$ & $\mathrm{C} 12-\mathrm{N} 3-\mathrm{C} 13-\mathrm{C} 14$ & $83.59(19)$ \\
$\mathrm{C} 6-\mathrm{N} 1-\mathrm{C} 7-\mathrm{C} 8$ & $1.8(2)$ & $\mathrm{N} 3-\mathrm{C} 13-\mathrm{C} 14-\mathrm{C} 15$ & $63.83(18)$ \\
$\mathrm{C} 6-\mathrm{N} 1-\mathrm{C} 7-\mathrm{C} 9$ & $-177.94(14)$ & $\mathrm{C} 13-\mathrm{C} 14-\mathrm{C} 15-\mathrm{C} 16$ & $171.75(15)$ \\
\hline
\end{tabular}

Symmetry codes: (i) $-x,-y,-z+1$; (ii) $-x+1,-y,-z+1$; (iii) $x-1, y, z$; (iv) $x+1, y, z$; (v) $-x+2,-y+1,-z+2$; (vi) $-x+2,-y+1,-z+1$; (vii) $-x+1,-y+1,-z+1$; (viii) $-x,-y,-z$.

Hydrogen-bond geometry $\left(\AA,{ }^{\circ}\right)$

$\mathrm{Cg} 1$ is the centroid of the $\mathrm{N} 3-\mathrm{N} 5 / \mathrm{C} 11 / \mathrm{C} 12$ ring.

\begin{tabular}{lllll}
\hline$D-\mathrm{H}^{\prime} \cdots A$ & $D-\mathrm{H}$ & $\mathrm{H} \cdots A$ & $D \cdots A$ & $D-\mathrm{H}^{\cdots} A A$ \\
\hline $\mathrm{C} 12-\mathrm{H} 12 \cdots \mathrm{N} 4^{\mathrm{iii}}$ & $0.954(19)$ & $2.419(19)$ & $3.2282(19)$ & $142.4(15)$ \\
$\mathrm{C} 2-\mathrm{H} 2 \cdots C g 1^{\mathrm{vii}}$ & $0.966(18)$ & $2.986(19)$ & $3.642(1)$ & $126.3(14)$
\end{tabular}

Symmetry codes: (iii) $x-1, y, z$; (vii) $-x+1,-y+1,-z+1$. 